\title{
VALIDEZ DEL TEST DE SALTO PARA LA VALORACIÓN DEL RENDIMIENTO ANAERÓBICO Y LA ASIMETRÍA EN EL CICLISMO DE ALTO NIVEL
}

\section{VALIDITY OF THE JUMP TEST FOR ASSESSING THE ANAEROBIC \\ PERFORMANCE AND ASYMMETRY IN ELITE CYCLISTS}

Xabier Muriel, Centro Tecnificación Deportiva BPX de Tolosa, Tolosa [Gipuzkoa] • Jesús Cámara-Tobalina, Facultad de Ciencias de la Actividad Física y el Deporte Universidad del País Vasco/Euskal Herriko Unibertsitatea [UPV/EHU], Vitoria/Gasteiz [Araba] • Juan Ramón Fernández-López, Centro Público de Enseñanzas Deportivas KIROLENE, Departamento de Educación del Gobierno Vasco, Durango [Bizkaia] • Jesús G Pallarés, Laboratorio de Fisiología del Ejercicio, Facultad de Ciencias del Deporte, Universidad de Castilla la Mancha [Toledo].

Correspondencia: xamuot@gmail.com [Xabier Muriel Otegui]

Recepción abril 2012 • Aceptación junio 2012

\section{RESUMEN}

Este estudio tuvo como objetivos validar el test de salto con contramovimiento (CMJ) para estimar el rendimiento anaeróbico, así como para estudiar la asimetría en la aplicación de fuerzas entre la pierna izquierda y derecha en ciclistas de alto nivel. Todos los participantes realizaron un test Wingate (WAnT) de 30s en un cicloergómetro y un test CMJ con registros de fuerza y potencia en cada pierna. Los resultados del estudio indican que el tiempo de vuelo del CMJ correlaciona significativamente con la eficiencia mecánica y potencia media y máxima desarrollada en el WAnT $(r=.61-.80 ; \mathrm{P}<.05)$. No obstante, la asimetría entre piernas registrada en ambos test (CMJ vs. WAnT) no correlaciona significativamente. Estos resultados sugieren que el CMJ puede ser un test válido para estimar el rendimiento del metabolismo anaeróbico en ciclistas de élite, aunque no parece ser un test válido para detectar déficits de aplicación de fuerzas entre piernas.

Palabras clave: test Wingate, salto con contramovimiento, fuerza, potencia, déficit de fuerza.

\section{ABSTRACT}

This study aimed to validate the countermovement jump test (CMJ) to estimate the anaerobic performance, as well as to study the asymmetry in the power application between left and right legs in elite cyclists. All participants performed a 30s Wingate test (WAnT) on a cycle ergometer and a CMJ test with force and power records on each leg. The results indicate that the CMJ flight time correlated significantly ( $\mathrm{r}=.61-.80$, $\mathrm{P}<.05)$ with the mechanical efficiency as well as with the average and maximum WAnT power. However, the asymmetry between legs recorded in both test (CMJ vs. WAnT) did not significantly correlate. These results suggest that the CMJ can be a valid test to estimate the anaerobic metabolism performance in elite cyclists, although there seems not to be a valid test for detecting strength deficits in the forces applied between legs.

Key Words: Wingate test, countermovement jump, strength, power, strength deficit.

\section{INTRODUCCIÓN}

La mayoría de competiciones de ciclismo en ruta están englobadas dentro de las denominadas carreras de salida masiva. Éstas a su vez se diferencian en tres tipos de carrera en función de la orografía: las carreras llanas (CLL), de media montaña (CMM) y de alta montaña (CAM) (Faria, 1984; Jeukendrup, Craig y Hawley, 2000; Padilla et al., 2001), que en alto nivel se caracterizan todas ellas por exigir un elevada capacidad aeróbica (Atkinson, Davison, Jeukendrup y Passfield 2003; Lucia, Hoyos, Carvajal y Chicharro, 1999; Lucia, Hoyos y Chicharro, 2001; Mujika y Padilla 2001; Padilla, Mujika, Santisteban, Impellizzeri y Goiriena, 2008). Estimar la intensidad del esfuerzo en ciclismo mediante la frecuencia cardíaca puede llegar a tener índices de validez relativamente bajos. Por ejemplo, la respuesta de la frecuencia cardíaca ante las demandas de carga externa (i.e., el conjunto de actividades que constituyen la unidad de entrenamiento) como las que ocurren en ciclismo con los cambios de ritmo, sprints, etc., ha mostrado una respuesta retardada (Ebert, Martin, Stephens y Withers, 2006). Además, puede darse el caso que para un mismo valor promedio de frecuencia cardíaca en un periodo de tiempo concreto, el desarrollo de potencia haya sido muy diferente. Es más, por el efecto de la deriva cardíaca, para una misma carga externa constante, la frecuencia cardíaca puede variar considerablemente en el tiempo, posiblemente debido a la deshidratación y aumento de la temperatura central (Achten, Venables y Jeukendrup, 2003; Ebert et al., 2006).

Estas circunstancias han propiciado el desarrollo de nuevos instrumentos y metodologías para el análisis y estudio de la intensidad de entrenamiento y competición en ciclismo. Entre estos novedosos sistemas destaca el análisis de la potencia desarrollada (i.e., producto de la fuerza aplicada en el pedal por el ciclista y la velocidad a la que se desplaza la biela), cuya característica fundamental es que permite medir intensidades muy altas desarrolladas en cortos periodos de tiempo, que hasta la fecha con el análisis de la frecuencia cardíaca no se habían podido registrar (Vogt et al., 2006). Mediante esta nueva forma de análisis, se ha podido observar que la capacidad de desarrollar altos picos de potencia en cortos periodos de tiempo se tornan determinantes en las etapas de CLL y CMM para la consecución del éxito, al contrario que las etapas de CAM, donde lo determinante es mantener constante una elevada potencia (Padilla et al., 2008; Vogt et al., 2007). En las etapas de CLL o CMM suele haber muchos cambios de ritmo en busca de las escapadas, ascensiones cortas y finales de etapa con sprint masivos, que implican la necesidad de producir altas potencias en corto espacio de tiempo (Faria, Parker y Faria, 2005; Fernández-García et al., 2000). Esta capacidad de desarrollar altos picos de potencia en cortos periodos de tiempo está condicionada en gran medida por la capacidad anaeróbica del ciclista (entendida como el sistema metabólico no dependiente del oxígeno) (Faria et al., 2005), por lo que la obtención de energía proveniente de estas rutas metabólicas anaeróbicas se convierte en uno de los principales factores determinantes del rendimiento y el éxito del ciclista (Faria et al., 2005; Jeukendrup et al., 2000; Vogt et al., 2007).

Hasta la aparición y desarrollo de los potenciómetros, la capacidad anaeróbica no se ha podido medir con razonables índices de validez dentro de la propia competición de ciclismo en ruta (Vogt et al., 2007), aunque en condiciones de laboratorio la potencia de pedaleo se ha estado registrando desde hace varias 
décadas (Bar-Or, 1987; Craig, Pyke y Norton, 1989; Szogy y Cherebetiu, 1974). En este sentido, el test Wingate (WAnT) es la valoración más utilizada para estimar la capacidad anaeróbica en laboratorio (Arslan, 2005; Bar-Or, 1987; Inbar, Bar-Or y Skinner, 1996). Este test consiste en un esfuerzo máximo de 30 segundos en cicloergómetro, sin ningún tipo de distribución del esfuerzo, y que permite estimar la capacidad y potencia anaeróbica del atleta (Bar-Or, 1987; Faria et al., 2005). No obstante, desde su primera publicación en 1977 (Bar-Or 1977), la validez de este test ha sido cuestionada en diferentes ocasiones (Beneke, Pollmann, Bleif, Leithauser y Hutler, 2002; Calbet, De Paz, Garatachea, Cabeza de Vaca y Chavarren, 2003; Dotan, 2006), así como su posible aplicación en otros deportes cíclicos o acíclicos que se alejen del pedaleo (Komi y Bosco, 1978; Rusko, Nummela y Mero, 1993; Sands, McNeal, Ochi, Urbanek, Jemni y Stone, 2004). Estudios posteriores han descrito diferentes modificaciones de los protocolos del WAnT que parecen maximizar los índices de reproducibilidad de esta valoración (Coso y Mora-Rodríguez, 2006; Laurent, Meyers, Robinson y Green, 2007; MacIntosh, Rishaug \& Svedahl, 2003; Neville, Pain, Kantor \& Folland, 2010).

Desde sus orígenes (Bar-Or, 1987), las principales variables de estudio en un test WAnT son: i) Pico de Potencia Máximo (i.e. valor máximo de potencia alcanzado en el test, generalmente dentro de los primeros $5 \mathrm{~s}$ ), ii) Potencia Media (i.e., valor promedio de potencia desarrollado en los $30 \mathrm{~s}$ ), y iii) Porcentaje o Índice de Fatiga (i.e., valor de Potencia Pico dividido por el valor de Potencia Mínima del test). Actualmente todavía se siguen utilizando estas tres variables para estimar el rendimiento anaeróbico del deportista, pero gracias a los recientes avances tecnológicos de los cicloergómetros se puede analizar cada una de estas variables con una mayor sensibilidad (i.e., frecuencia de muestreo) y diferenciando el trabajo y la potencia realizada por cada pierna. Varías casas comerciales han fabricado cicloergómetros que posibilitan medir el momento de fuerza (i.e., torque) de cada biela en todo el ciclo de la pedalada. Este desarrollo tecnológico permite medir las mismas variables que la perspectiva clásica del test WAnT pero analizando independientemente cada biela, y con ello el índice de asimetría entre ambas piernas, es decir, el posible déficit de aplicación de potencia de una pierna respecto a la otra (Carpes, Diefenthaeler, Bini, Stefanyshyn, Faria y Mota, 2011; Carpes, Rossato, Faria y Bolli Mota, 2007; Carpes, Mota y Faria, 2010). Este análisis de asimetría se ha mostrado efectivo para prevenir lesiones (Cohen, 1993; Impellizzeri et al., 2007) y optimizar la eficiencia de pedaleo (Carpes et al., 2007).

A pesar de que diferentes trabajos han mostrado índices razonables de validez y reproducibilidad del WAnT, en la actualidad este test ha reducido su presencia en los programas de valoración real del ciclista de medio y alto nivel debido principalmente al esfuerzo físico y mental extremo que requiere su ejecución (Coso y Mora-Rodríguez, 2006; Laurent et al., 2007). Además, el clásico WAnT de laboratorio reduce notablemente la especificidad de la prueba, ya que las dimensiones y los ajustes de la propia bicicleta de competición del ciclista son difíciles de reproducir en estos cicloergómetros de laboratorio. Además, las oscilaciones laterales producidas durante el ciclismo en carretera no se pueden simular sobre el cicloergómetro. Por todo ello surge la necesidad de proponer un test que permita evaluar la capacidad anaeróbica del ciclista, con una instrumentación de mayor portabilidad, así como con una exigencia física menos agónica, que no retrase en exceso la recuperación del deportista para poder continuar con el proceso de valoración o entrenamiento.

Diversos autores han encontrado correlaciones significativas entre los resultados de otras pruebas o test físicos y los resultados del WAnT. En este sentido, la batería de test de saltos de Bosco (Bosco, Luhtanen y Komi, 1983) es otra de las valoraciones más comunes y estándares para determinar la capacidad anaeróbica de los deportistas, aunque recientemente la correlación entre los resultados de los test de salto de Bosco y el WAnT se ha puesto en entredicho en deportistas no entrenados en la técnica del salto (Sands et al., 2004). Igualmente, Inbar y cols., (1996) observaron correlaciones significativas entre los resultados del WAnT y otros índices del metabolismo anaeróbico como la concentración de lactato después del esfuerzo. Diferentes variables neuromusculares como la fuerza explosiva y la fuerza isométrica también han mostrado correlaciones significativas con los datos del WAnT en jóvenes atletas (Arslan, 2005). No obstante, muy pocos de estos estudios fueron realizados con deportistas altamente entrenados y en ningún caso con ciclistas de élite. Igualmente, para el conocimiento de los autores, ninguna publicación ha correlacionado la asimetría del salto vertical (Impellizzeri et al., 2007) y la asimetría de piernas que podemos medir en los WAnT actualmente.

Estos hallazgos sugieren que los test de saltos de Bosco pueden ser una alternativa válida para evaluar el rendimiento anaeróbico en ciclistas, además de ser un test práctico para realizar en cualquier instalación y menos exigente físicamente. No obstante, su validez en ciclistas altamente entrenados y su correlación con la asimetría del miembro inferior durante el test WAnT todavía no están descritas. Por todo ello, los objetivos de este estudio fueron i) determinar la relación que existe entre los resultados del test WAnT de $30 \mathrm{~s}$ en cicloergómetro con los resultados del test de salto con contramovimiento y ii) determinar la relación que existe entre los resultados de asimetría entre piernas derecha e izquierda del test WAnT y los del índice de asimetría entre piernas del test de salto con contramovimiento. Nuestra hipótesis fue que, tomando el test WAnT como referencia o patrón, el salto con contramovimiento no es una prueba válida para estimar el rendimiento anaeróbico de ciclistas del ato nivel, seguramente debido a la poca experiencia que presentan estos deportistas con las técnicas de salto, aunque sí permite detectar las mismas asimetrías en la aplicación de fuerzas que ocurren durante el pedaleo en ciclistas de élite.

\section{MÉTODO}

\section{Participantes}

Para este estudio fueron seleccionados 11 ciclistas federados de las categorías junior y senior. Tres de los participantes ostentaban el título de campeón autonómico de ciclismo en ruta y 6 de ellos habían sido ganadores de competiciones a nivel nacional. La edad media de los ciclistas era de 21,0 $\pm 2,9$ años, y en la última temporada (octubre-septiembre del 2010) los participantes habían realizado una media de $16.454 \pm 6.088$ kilómetros, considerándose por ello a todos los participantes como sujetos muy entrenados y de alto nivel. Las características físicas de los ciclistas fueron: edad 21,0 $\pm 2,9$ años; masa corporal $67,7 \pm 5,8 \mathrm{~kg}$; talla $178,3 \pm$ $6,1 \mathrm{~cm}$; Sumatorio de 6 pliegues $48,4 \pm 12,9 \mathrm{~mm}$; \% Graso 7,7 $\pm 1,4$ $\%$.

\section{Diseño y Procedimientos}

Antes de comenzar la fase experimental, todos los participantes fueron informados en detalle sobre los procedimientos del estudio y sus posibles riesgos y beneficios para su preparación. Este estudio se realizó de acuerdo a la declaración de Helsinki y se obtuvo el consentimiento informado por escrito de todos los participantes antes de comenzar la fase experimental.

Composición corporal. Las medidas antropométricas practicadas fueron: talla, masa corporal, pliegues cutáneos (tríceps, subescapular, suprailíaco, abdominal, muslo anterior, pierna medial, supraespinal y bíceps braquial). Todas ellas fueron tomadas por el mismo investigador experimentado de acuerdo con las directrices de la International Society for the Advancement of Kineanthropometry (ISAK) (Marfell-Jones, Olds, Stewart y Carter, 2006). El porcentaje graso y la masa libre de grasa se estimaron empleando la fórmula de Carter y Yuhasz (1984).

Salto con contra movimiento (CMJ). El test de salto con contramovimiento (CMJ) es un test de salto totalmente estandarizado y utilizado en multitud de trabajos científicos desde que fue descrito por Carmelo Bosco en la década de los 80 (Bosco, Luhtanen P \& Komi, 1983) para la valoración del rendimiento físico del tren inferior. El movimiento durante la realización del CMJ se caracteriza 
por ser un gesto acíclico, de cadena cinética compleja, en el que ambas piernas ejecutan la acción concéntrica de empuje simultáneamente. Además, el test CMJ es una valoración pesodependiente, ya que el participante tiene como única resistencia externa la de su propia masa corporal. Para este estudio, todos los participantes realizaron dos sesiones de familiarización con el protocolo del test (Bosco, 1994). En el transcurso de estas dos sesiones de familiarización los ciclistas realizaron entre 20 y 25 repeticiones del salto $\mathrm{CMJ}$. El equipo investigador mantuvo en todo momento un feedback con el participante para corregir la técnica de salto, y de esta forma evitar cualquier efecto del aprendizaje de la técnica sobre la reproducibilidad de la medida durante el estudio. La componente vertical de la fuerza de reacción del suelo se registró durante todas las fases de salto. Para ello se utilizaron dos plataformas de fuerza (Kirolene, Durango y Útiles y Máquinas, Bilbao) a una frecuencia de $500 \mathrm{~Hz}$ para que participantes colocasen tanto en la fase de despegue como de aterrizaje un pie en cada una de las plataformas (i.e., derecha e izquierda). En bipedestación y con las manos en las caderas en todo momento, el participante realizó una flexo-extensión de caderas y rodillas a la máxima velocidad de ejecución posible. Los sujetos fueron instruidos para mantener la misma posición de cadera, rodillas y tobillos en el despegue y aterrizaje. En función de la variable tiempo de vuelo, se registró para su posterior análisis la media de los 3 mejores saltos de una serie de 5 repeticiones. Las fuerzas de reacción registradas por ambas plataformas (pie derecho e izquierdo), se almacenaron para su posterior análisis de los registros y/o estimaciones de las siguientes variables: peso del sujeto $(\mathrm{N})$, tiempo de vuelo $(\mathrm{s})$, altura del centro de gravedad $(\mathrm{m})$, velocidad de despegue $(\mathrm{m} \cdot \mathrm{s}-1)$, pico mínimo de fuerza $(\mathrm{N})$, pico máximo de fuerza $(\mathrm{N})$ e índice de asimetría (Stacoff, Diezi, Luder, Stüsi, y Krames-de-Quervain, 2005) del pico mínimo y máximo de fuerza.

Test Wingate (WAnT): Una vez realizados los test de salto, y tras una recuperación completa de $30 \mathrm{~min}$, todos los participantes realizaron el test WAn'T de pedaleo de $30 \mathrm{~s}$ en cicloergómetro (BarOr, Dotan \& Inbar, 1977). Esta valoración se caracteriza por ser un gesto cíclico de cadena cinética sencilla, en la que ambas piernas ejecutan la acción concéntrica de empuje de forma alterna. Además, el test WAnT es una valoración peso-no- dependiente, ya que la resistencia a vencer puede configurarse y manipularse desde el propio cicloergómetro, y la masa corporal del participante reposa sobre el sillón, manillar y pedales. $\quad$ El test WAnT comenzó con 8 minutos de calentamiento a $80 \mathrm{rpm}$, seguidos de $30 \mathrm{~s}$ de esfuerzo máximo, sin levantarse del sillín y sin ningún tipo de descanso entre el calentamiento y el esfuerzo máximo. El test se realizó en el cicloergómetro (Lode Excalibur Sport, The Netherlands), cuyo software asociado calculaba y programaba la resistencia o carga de pedaleo en función a la masa del sujeto $(7,5$ $\%$ de la masa corporal), tanto para el calentamiento, como para el esfuerzo máximo. Todos los participantes realizaron una fase de familiarización con el cicloergómetro antes de realizar la prueba. A su vez, antes del inicio de la misma, el cicloergómetro fue ajustado (en altura y retroceso del sillín y manillar) conforme a las medidas de las propias bicicletas de competición de cada ciclista. El test se realizó con las propias botas y pedales de competición de cada ciclista fijados mediante calas. Antes del comienzo de cada test, se calibró el cicloergómetro siguiendo el protocolo validado del fabricante. El software del cicloergómetro registró las siguientes variables de cada una de las piernas así como de la suma de ambas: revoluciones por minuto (ciclos $\cdot \min -1)$, pico de potencia (W), potencia media (W), potencia mínima (W) e índice de fatiga. Se normalizaron a su vez todos los registros de potencia según la masa corporal (PP/MC) y masa libre de grasa del sujeto (PP/MLG) (W·kg-1). ). La eficiencia mecánica (EM) se calculó mediante la siguiente fórmula:

Fórmula 1: eficiencia mecánica medida según la Lode Excalibur:

$$
E M=100 x \frac{\int_{0}^{2 \Pi} T_{\text {тот }}(\theta)}{\int_{0}^{2 \Pi} T_{\text {E }}(\theta)}
$$

, donde _ es el ángulo de la biela, TTOT es la suma del momento de fuerza propulsivo y resistivo de ambas piernas, y TE es la suma del momento de fuerza propulsivo.

Análisis Estadístico: Para el análisis de las características de los sujetos se emplearon métodos descriptivos estándar. Para la determinación de la relación entre las variables del test Wan'T y el test CMJ en ambas piernas se empleó el análisis de correlación Pearson, una vez confirmados los supuestos de normalidad y homocedasticidad. El supuesto de normalidad se analizó con el estadístico Kolmogorov-Smirnov y Shapiro-Wilk. El supuesto de homocedasticidad con la prueba de Levene. En todo caso se consideró un nivel de significación estadística del 5\% ( $\leq .05)$.

\section{RESULTADOS}

La Tabla 1 muestra la media y desviación estándar de los principales resultados en valor absoluto de los test CMJ y WAnT.

Tabla 1. Resumen de los principales resultados obtenidos en las valoraciones de salto con contramovimiento (CMJ) con plataformas de fuerza y del test Wingate (WAnT) en cicloergómetro con registros de potencia en cada pierna.

\begin{tabular}{|l|l|c|c|c|}
\hline & & & & \\
\hline CMJ & & & & \\
\hline & Tiempo de vuelo (s) & 0.481 & \pm & 0.042 \\
\hline & Altura de salto (cm) & 29.0 & \pm & 5.0 \\
\hline & Pico de fuerza I + D (N) & 1715 & \pm & 252 \\
\hline & Pico de fuerza I (N) & 843 & \pm & 120 \\
\hline & Pico de fuerza D (N) & 898 & \pm & 168 \\
\hline WAn'T & & & & \\
\hline & & & & \\
\hline & Potencia media máxima I (W) & 1046 & \pm & 159 \\
\hline & Potencia media máxima D (W) & 1090 & \pm & 156 \\
\hline & Potencia Pico I (W) & 1487 & \pm & 226 \\
\hline & Potencia Pico D(W) & 1525 & \pm & 234 \\
\hline & Índice de Eficiencia & 94 & \pm & 3 \\
\hline & RPM (ciclos · min-1) & 136 & \pm & 9 \\
\hline
\end{tabular}


Tabla 2. Resultados de las principales correlaciones entre las variables estudiadas en el test Wingate y el test CMJ para las piernas derecha e izquierda.

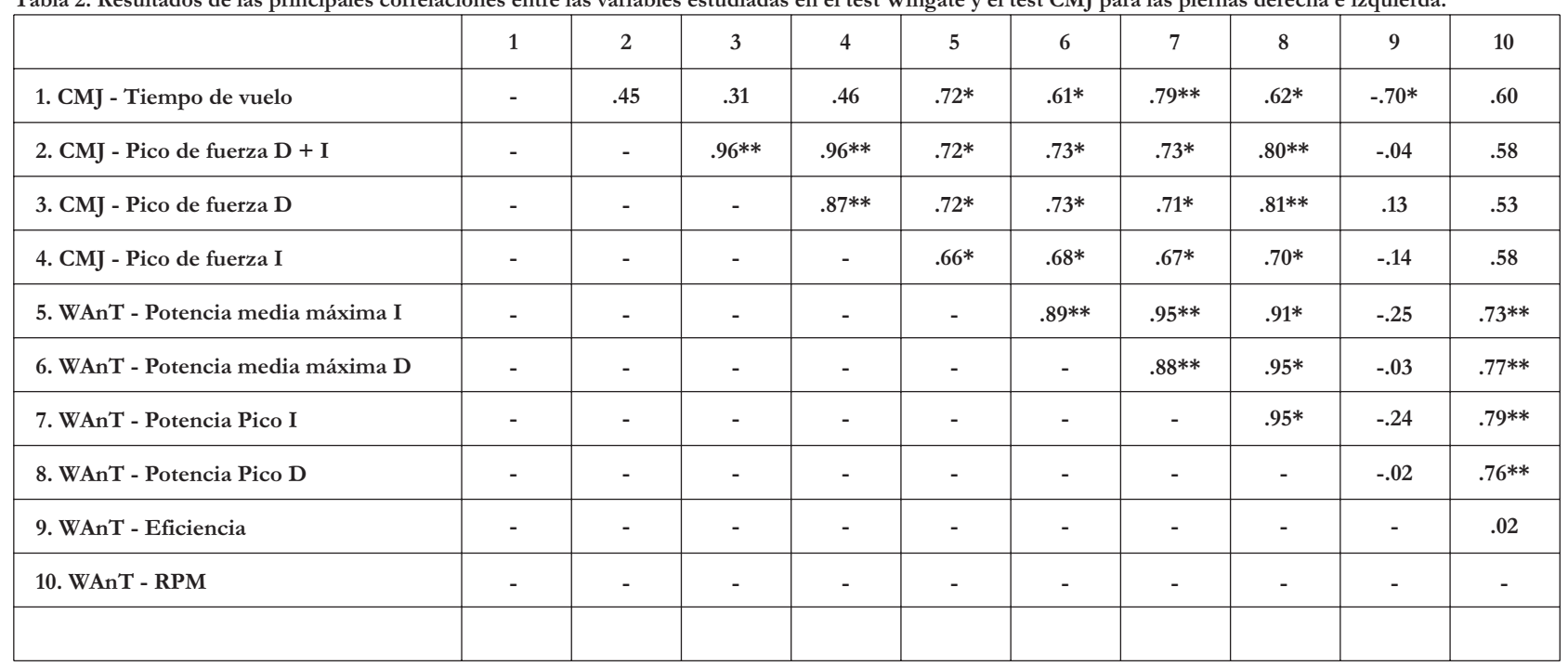

CMJ: test de salto con contramovimiento; WAnT: test Wingate; D: derecha; I: izquierda; RPM: revoluciones por minuto; * $\mathrm{P}<.05$. $* * \mathrm{P}<.001$.

En relación a los resultados de los test CMJ, la únicas variables que mostraron correlación significativa $(\mathrm{P}<.01)$ con alguna variable del test WAnT fueron el tiempo de vuelo y el pico de fuerza generado en las plataformas de ambas piernas. Las correlaciones con el resto de variables, aunque en algunos casos muestran tendencia, no son estadísticamente significativas (Tabla 2). Las Figura 1 muestra la correlación estadísticamente significativa que existe entre la Potencia Pico por pierna, tanto derecha (A) como izquierda (B) en el WAn'T, y el tiempo de vuelo de los saltos CMJ.

Así mismo, la Figura 2 muestra la correlación estadísticamente significativa que existe entre la Potencia Media Máxima por pierna, tanto derecha (A) como izquierda (B) en el WAnT, y el tiempo de vuelo de los saltos CMJ.

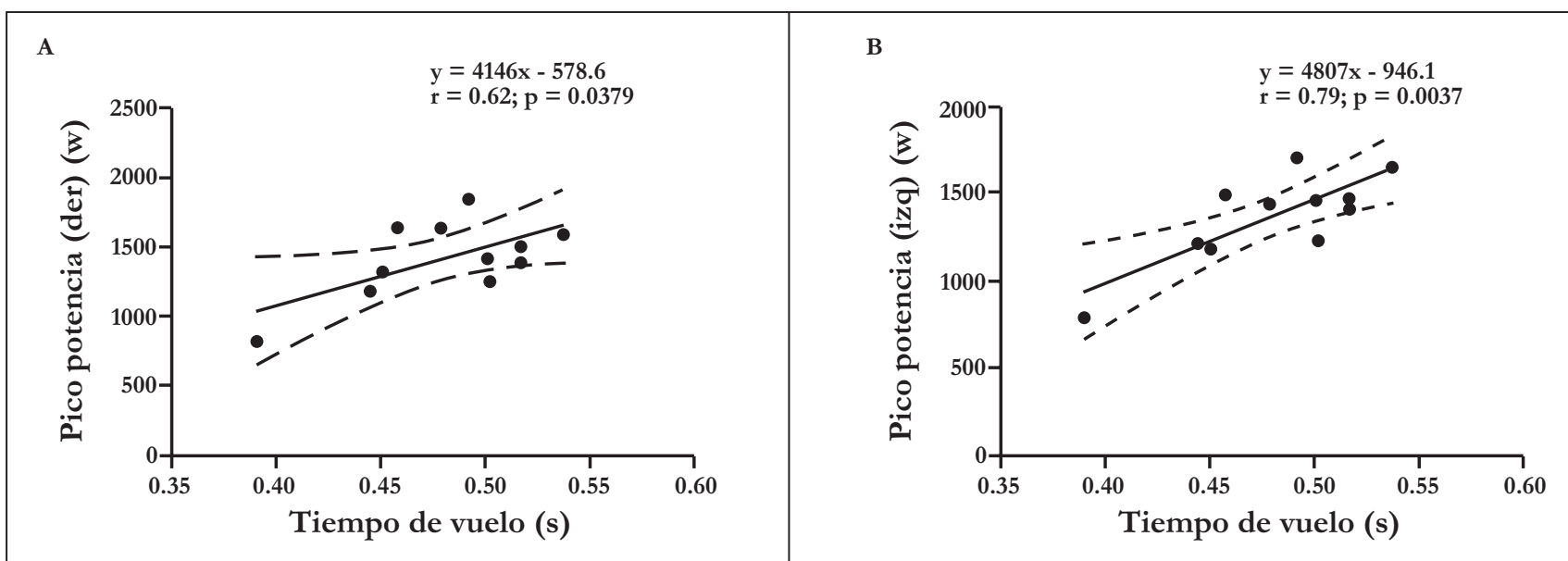

Figura 1. Relación entre tiempo de vuelo en CMJ y la Potencia Pico para la pierna derecha (A) y pierna izquierda (B) en el WAnT.

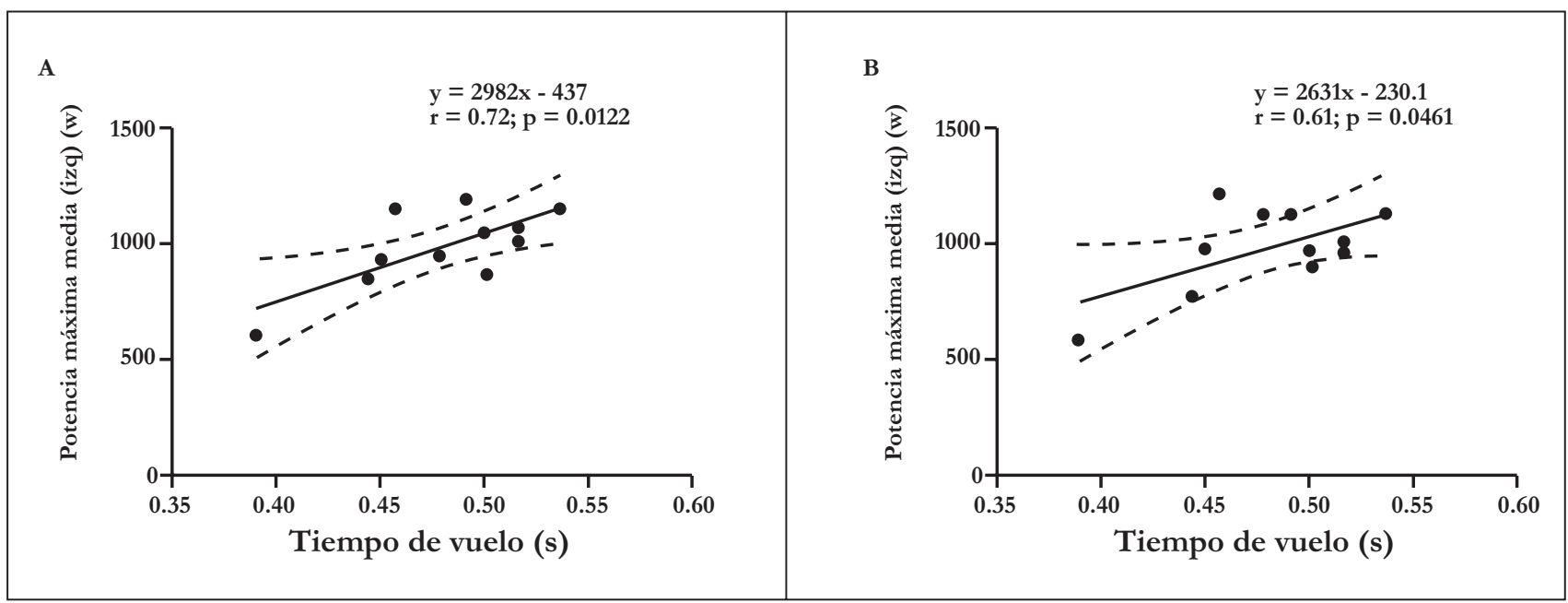

Figura 2. Relación entre WAnT Potencia Media Máxima por pierna, tanto derecha (A) como izquierda (B) y el tiempo de vuelo de los saltos CMJ. 
La eficiencia mecánica del WAnT, calculado por el software del propio cicloergómetro, y el tiempo de vuelo en CMJ también mostraron una correlación significativa (Figura 3).

Por su parte, el pico de fuerza de ambas piernas obtenido en el test CMJ mostró correlaciones significativas, tanto con la potencia media máxima, como con la potencia pico de ambas piernas en el WAnT (rango $\mathrm{r}=.66-.81 ; \mathrm{P}<.05$ ). En cuanto a los datos de asimetría, no se han encontrado correlaciones estadísticamente significativas entre los índices de asimetría del CMJ y los índices de asimetría del Wingate en ninguna de las variables estudiadas.

\section{DISCUSIÓN}

Los objetivos de este estudio fueron, en primer lugar determinar la relación que existe entre los valores de potencia anaeróbica estimados por los dos principales test empleados en la literatura internacional para valorar el rendimiento anaeróbico del tren inferior: $1^{\circ}$ test CMJ (test peso-dependiente, con extensión simultánea del tren inferior y áciclico) y $2^{\circ}$ test WAnT en cicloergómetro (test peso-no-dependiente, con flexo-extensión alterna del tren inferior y cíclico) en una muestra de ciclistas de alto nivel. En segundo lugar, este estudio tuvo como objetivo determinar la relación existente entre los resultados de asimetría entre ambas piernas de estas dos valoraciones.

Los principales hallazgos del estudio fueron que el tiempo de vuelo del test CMJ y los valores de potencia pico y potencia media máxima del WAn'T correlacionan significativamente en ciclistas de alto nivel. Así mismo, la eficiencia mecánica del test Wingate, un indicador para caracterizar la relación entre las fuerzas propulsivas y resistivas perpendiculares a la biela durante todo el ciclo de la pedalada, muestra correlaciones significativas con el tiempo de vuelo del test CMJ. Estos resultados son importantes y únicos, ya que para nuestro conocimiento, no se ha descrito con anterioridad esta relación en sujetos de esta modalidad deportiva ni tampoco en este nivel de rendimiento. Las aplicaciones prácticas de este hallazgo pueden ser de gran ayuda para los técnicos y científicos relacionados con este tipo de deportistas, ya que, a la luz de estos resultados, el rendimiento del metabolismo anaeróbico en ciclismo puede estimarse tanto con un test acícilico y de muy corta duración (i.e., $\mathrm{CMJ}$ ), como con el test cíclico WAn'T de piernas de $30 \mathrm{~s}$, valoración esta última que genera una altísima acidosis metabólica, un descenso muy importante de los sustratos energéticos asociados a esta ruta metabólica, y que por consiguiente requiere de una recuperación de la fatiga más prolongada (Pallarés \& MoránNavarro, 2012). Por otro lado, del estudio de asimetrías o déficits de fuerza y potencia que ocurren entre piernas para los test WAn'T y CMJ se desprende que no existe relación entre ambas metodologías, por lo que parece recomendable que los ciclistas continúen realizando el estudio de asimetrías en su propio gesto técnico de entrenamiento y competición como es el pedaleo.

Los resultados de la altura de vuelo alcanzada en el test CMJ por los ciclistas de este estudio $(29.0 \pm 5.0 \mathrm{~cm})$ son notablemente inferiores a los descritos en estudios previos que tomaron como sujetos experimentales a varones de alto nivel en diferentes modalidades deportivas en los que la capacidad de salto era una factor determinante como en voleibol $(44.4 \pm 4.7 \mathrm{~cm}$ Riggs y Sheppard, 2009) o en balonmano (46.8 \pm 7.0 - Gorostiaga, Granados, Ibáñez e Izquierdo, 2005). Por el contrario, los valores de potencia pico $(1558 \pm 222 \mathrm{~W})$ y en especial los de potencia pico relativo a la masa corporal $(22.7 \pm 2.4 \mathrm{~W} \cdot \mathrm{kg}-1)$ registrados en este estudio para el test WAnT son muy superiores a los registrados en deportistas de otras especialidades como en tenistas $(772 \pm 94 \mathrm{~W}$; 11.6 $\pm 0.8 \mathrm{~W} \cdot \mathrm{kg}-1$ - Zagatto, Papoti y Gobatto, 2008) o jugadores de rugby (912 $\pm 152 \mathrm{~W} ; 10.7 \pm 1.6 \mathrm{~W} \cdot \mathrm{kg}-1$ - McIntyre \& Hall, 2005), y muy similares a los descritos por otros autores con ciclistas de este mismo nivel competitivo $(1547 \pm 128 \mathrm{~W}$ y $20.6 \pm 1.3 \mathrm{~W}$. kg-1 - Calbet et al., 2003; $1599 \pm 370$ W - Zabala et al., 2010). Al igual que en los resultados del presente trabajo, investigaciones previas han registrado correlaciones significativas entre los valores de potencia máxima y media del test WAnT y diferentes variables neuromusculares medidas en test de saltos como la fuerza explosiva o el tiempo de vuelo (Arslan, 2005; Fatouros et al., 2011), aunque en la mayoría de ellos se tomaron como participantes a sujetos no entrenados, y en ningún caso a ciclistas de ningún nivel competitivo. Además de estas diferencias en la muestra, los instrumentos que estos investigadores han empleado para conocer el rendimiento de las variables neuromusculares relacionadas con los test de salto (e.g., plataforma de contactos, dinamómetro con galga extensiométrica, etc.) y el WAn'T (i.e., cicloergómetros sin potenciómetros que estiman la potencia con frecuencias de muestreo muy bajas) poseen unos niveles de precisión, validez y reproducibilidad inferiores a los de las plataforma de fuerzas y el cicloergómetro que se han empleado en este estudio.

El test Wingate de piernas se viene empleando desde hace 4 décadas como principal valoración de la potencia y la capacidad anaeróbica del deportista, independientemente de su especilidad deportiva y de su patrón motor. Para el ciclismo, esta valoración cumple a la perfección con el princpio de especificidad, ya que permite valorar el rendmiento anaeróbico sobre el propio gesto técnico de competición. Aplicar el principio de especificidad resulta fundamental para optimizar el rendimiento en cualquier modalidad deportiva. La especificidad de los estímulos de entrenamiento sobre las adaptaciones que estos producen ha sido extensamente estudiada en la literatura científica (García-Pallarés, Sánchez-Medina, Carrasco, Díaz e Izquierdo, 2009; Gibala et al.,

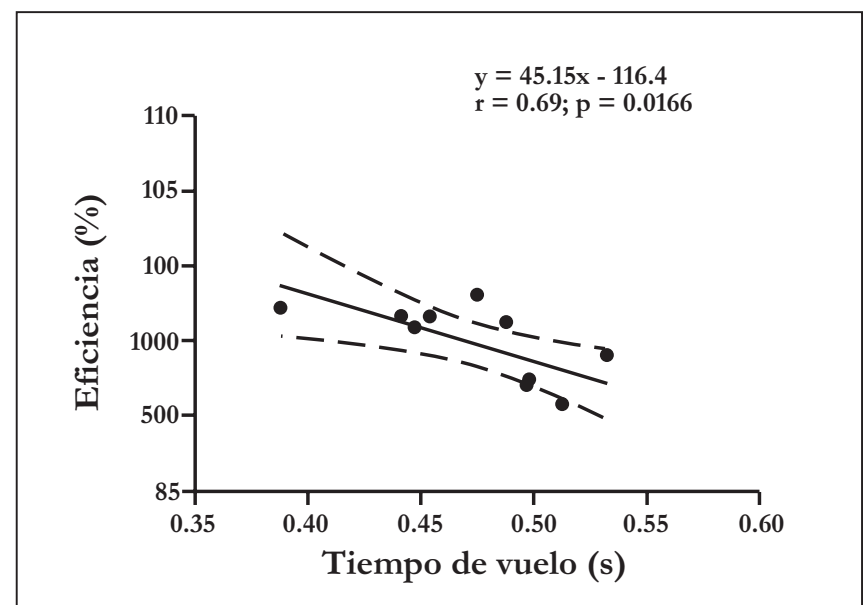

Figura 3. Relación entre tiempo de vuelo en CMJ y la eficiencia mecánica del WAnT. 
2006; Helgerud, et al., 2007), así como la idoneidad de maximizar el tiempo de preparación que realiza el deportista con el propio gesto técnico o patrón motor de competición (Fernhall y Kohrt, 1990; Navarro y Verdugo, 2007; Verchoschanskij, 1990). Multitud de autores han aplicado estos hallazgos al campo de la valoración y testaje del rendimiento deportivo, según los cuales las valoraciones del deportista se deben realizar, no sólo en los mismos gestos técnicos de competición, sino que, en la medida de lo posible, se deben realizar con el mismo tipo de contracción muscular, velocidad de contracción, sustrato energético, ruta metbólica, etc. (GonzálezBadillo y Ribas-Serna, 2002; Navarro y Verdugo, 2007). No obstante, tal y como se ha comentado, las características del protocolo del WAnT original de $30 \mathrm{~s}$ tiene asociado multitud de problemas relacionados principalmente con la fatiga extrema que genera en el participante, y por ello con el tiempo que es necesario guardar tras este test para poder realizar otro estímulo de entrenamiento o valoración con una recuperación completa (Bar-Or et al., 1977; Bar-Or, 1987). Por ello, este estudio ha tratado de validar el test CMJ que, a pesar de inclumplir el principio de especificidad, permite valorar la condición anaeróbica de cislitas de élite sin que el esfuerzo realizado impida continuar inmediatamente con el programa de valoraciones o con una sesión de entrenamiento.

Tratando de mantener el pricipio de especificidad, estudios previos han propuesto diferentes modificaciones del test WAnT que permiten incrementar sus niveles de reproducibilidad y validez (MacIntosh et al., 2003; Neville et al., 2010), pero sobre todo existe un grupo reducido de estudios que han validado modificaciones del test WAnT para evaluar la potencia anaeróbica del deportista con una duración menor a $5 \mathrm{~s}$, evitando de esta forma la fatiga extrema que genera un test WAnT all-out de $30 \mathrm{~s}$ (Coso y MoraRodríguez, 2006; Martin, Wagner y Coyle, 1997). Estas modificaciones propuestas al protoclo del WAnT original, además de aumentar la calidad de la medida (i.e., reproducibilidad y validez), mantienen la especificidad del gesto técnico del pedaleo, y por lo tanto es adecuado para aplicarlo en ciclistas o triatletas. No obstante, los recursos necesarios para realizar tanto el WAnT original de 30 s como el WAnT de 4 ó $5 \mathrm{~s}$, donde es necesario un cicloergómetro que mida la potencia de forma directa con potenciómetros o trasductores lineales de velocidad y posición, y a una frecuencia de muestreo mayor (e.g., $1 \mathrm{~Hz}$ WAnT 30 s vs. $500 \mathrm{~Hz}$ WAnT de 4-5 s) son muy costosos, delicados, difícilmente portables y requieren de una gran capacidad de configurar sus dimensiones a las medidas antropométricas de cada ciclista. Por ello el principal objetivo de este estudio ha sido realizar la validación de un test de salto que, a pesar de perder especificidad en el gesto técnico respecto al pedaleo, mantuviese los índicies de validez aceptables, al tiempo que se simplifican los protocolos, se reduce el coste económico del instrumento y se facilita la portabilidad del mismo. Además, los resultados de este estudio indican que la variable tiempo de vuelo del test CMJ correlaciona significativamente con los valores de eficiencia mecánica y potencia media y máxima del test WAnT de $30 \mathrm{~s}$ en ambas piernas. Estos hallazgos sugieren que este mismo protocolo de valoración con el test de salto CMJ puede llegar a realizarse sin la plataforma de fuerzas como instrumento de registro, ya que podría ser suficiente con una plataforma de contactos que permita conocer el tiempo de vuelo de forma mucho más económica, portable y accesible.

Por otro lado, a pesar de que el test de salto CMJ parece ser un test válido para estimar la potencia anaeróbica de los ciclistas, los resultados de este estudio refutan nuestra segunda hipótesis ya que nos indican que no permite detectar las asimetrías de aplicación de fuerza entre la pierna izquierda y derecha en ciclistas de alto nivel. En este sentido, es necesario destacar que actualmente existen en el mercado muy pocos cicloergómetros y potenciómetros capaces de medir la potencia generada por cada pierna a una velocidad de muestreo suficiente que permita detectar asimetrías. Estos pocos ergómetros, con marcas comerciales como Lode, SRM y Watt Bike, son muy costosos y poco accesibles a los técnicos relacionados con el ciclismo. Por ello este estudio trató de validar un test de salto con 2 plataformas de fuerzas capaces de registrar la aplicación de fuerzas independientemente en cada pierna, y de esta forma hacer más accesible a los ciclistas y sus técnicos la evaluación de las asimetrías. La validez de ambos gestos técnicos (i.e., pedalada y salto) para detectar asimetrías de aplicación de fuerza en el miembro inferior se ha estudiado y corroborado previamente por separado (Bra_i_, Supej, Stanislav, Ba_i_y _oh, 2010; Carpes et al., 2010; Impellizeri et al., 2007), a pesar de que hasta la fecha no se había establecido su correlación. Aunque la hipótesis de nuestro estudio fue que el test CMJ permitiría detectar asimetrías del tren inferior en ciclistas experimentados, la ausencia de correlación entre las asimetrías de ambas valoraciones (i.e., CMJ vs. WAnT) puede ser debida a la escasa experiencia previa de los participantes con la técnica de salto CMJ, y por el contrario al gran bagaje y experiencia que poseen estos ciclistas con el patrón motor del pedaleo.

En conclusión el tiempo de vuelo del test de CMJ correlaciona significativamente con las principales variables que estiman la potencia y capacidad anaeróbica del test WAnT de piernas de $30 \mathrm{~s}$. Estos resultados permiten a los técnicos o científicos relacionados con cualquier modalidad deportiva emplear el test CMJ para estimar el rendimiento del metabolismo anaeróbico, reduciendo notablemente la complejidad y la logística del test, al tiempo que se reduce los recursos y el coste de estas valoraciones. Además, este estudio muestra que no existe correlación entre la asimetría registrada por el potenciómetro en el pedaleo del WAnT con la asimetría detectada por las plataformas de fuerza con el test CMJ. Por ello, parece recomendable que la valoración de las asimetrías y déficits de aplicación de fuerzas en ciclistas y triatletas experiementados se continúe realizando en cicloergóemtros, manteniendo de esta forma la maxíma especificdad posible respecto del propio gesto del pedaleo.

\section{REFERENCIAS BIBLIOGRÁFICAS}

Achten, J., Venables, M. C., \& Jeukendrup, A. E. (2003). Fat oxidation rates are higher during running compared with cycling over a wide range of intensities. Metabolism,52(6), 747-752.

Arslan, C. (2005). Relationship between the 30 -second wingate test and characteristics of isometric and explosive leg strength in young subjects. Journal of Strength \& Conditioning Research, 19(3), 658-666.

Atkinson, G., Davison, R., Jeukendrup, A., \& Passfield, L. (2003). Science and cycling: current knowledge and future directions for research. Journal of Sports Science, 21(9), 767-787.

Bar-Or, O., Dotan, R., \& Inbar, O. (1977). A $30 \mathrm{~s}$ all out ergometry test: its reliability and validity for anaerobic capacity. Israel Journal of Medicine Sciences in Sports \& Exercise, 13, 326327.

Bar-Or, O. (1987). The Wingate anaerobic test. An update on methodology, reliability and validity. Sports Medicine, 4(6), 381394.

Beneke, R., Pollmann, C., Bleif, I., Leithauser, R. M., Hutler, M. (2002). How anaerobic is the Wingate Anaerobic Test for humans?. European Journal of Applied Physiology, 87(4-5), 388392.

Bosco, C. (1994) La valoración de la fuerza con el test de Bosco. Barcelona: Paidotribo.

Bosco, C., Luhtanen, P., \& Komi, P. V. (1983). A simple method for measurement of mechanical power in jumping. European Journal of Applied Physiology and Occupational Physiology, 50(2), 273-282.

Bra_i_, M, Supej, M., Stanislav, P., Ba_i_, P \&_oh, M. (2010). An investigation of the influence of bilateral deficit on the countermovement jump performance in elite sprinters. Kinesiology, 42(1), 73-81.

Calbet, J. A., De Paz, J.A., Garatachea, N., Cabeza de Vaca, S., \& Chavarren, J. (2003). Anaerobic energy provision does not limit Wingate exercise performance in endurance-trained cyclists. Journal of Applied Physiology, 94(2), 668-676.

Carpes, F. P., Diefenthaeler, F., Bini, R. R., Stefanyshyn, D. J., Faria, I. E., \& Mota, C.B. (2011). Influence of leg preference on bilateral muscle activation during cycling. Journal of Sports Science, 


\section{9(2), 151-159.}

Carpes, F. P., Mota, C. B., \& Faria, I. E. (2010). On the bilateral asymmetry during running and cycling - a review considering leg preference. Physical Therapy in Sport, 11(4), 136-142.

Carpes, F. P., Rossato, M., Faria, I. E., \& Bolli Mota, C. (2007). Bilateral pedaling asymmetry during a simulated $40-\mathrm{km}$ cycling time-trial. Journal of Sports Medicine and Physical Fitness, 47(1), 51-57.

Carter, J. E. L., \& Yuhasz, M. S. (1984). Skinfolds and body composition of Olympic athletes. In: Carter, J. E. L., editor. Physical structure of Olympic athletes. Part II: Kinanthropometry of Olympic athletes. Basel: Karger.

Cohen, G. C. (1993). Cycling injuries. Canadian Family Physician, 39, 628-632.

Coso, J. D., \& Mora-Rodríguez, R. (2006). Validity of cycling peak power as measured by a short-sprint test versus the Wingate anaerobic test. Applied Physiology, Nutrition and Metabolism, 31(3), 186-189.

Craig, N. P., Pyke, F. S., \& Norton, K. I. (1989). Specificity of test duration when assessing the anaerobic lactacid capacity of high-performance track cyclists. International Journal of Sports Medicine, 10(4), 237-242.

Dotan, R. (2006). The Wingate anaerobic test's past and future and the compatibility of mechanically versus electro-magnetically braked cycle-ergometers. European Journal of Applied Physiology, 98(1), 113-116.

Ebert, T. R., Martin, D. T., Stephens, B., \& Withers, R. T. (2006). Power output during a professional men's road-cycling tour. International Journal of Sports Physiology \& Performance, 1(4), 324-335.

Faria, E. W., Parker, D. L., \& Faria, I. E. (2005). The science of cycling: physiology and training - part 1. Sports Medicine, 35(4), 285-312.

Faria, I. E. (1984). Applied physiology of cycling. Sports Medicine. 1(3), 187-204.

Fatouros, I. G., Laparidis, K., Kambas, A., Chatzinikolaou, A., Techlikidou, E., Katrabasas, I., Douroudos, I., Leontsini, D., Berberidou, F., Draganidis, D., Christoforidis, C., Tsoukas, D., Kelis, S., \& Taxildaris, K. (2011). Validity and reliability of the single-trial line drill test of anaerobic power in basketball players. Journal of Sports Medicine and Physical Fitness, 51(1), 33-41.

Fernhall, B., \& Kohrt, W. (1990). The effect of training specificity on maximal and submaximal physiological responses to treadmill and cycle ergometry. Journal of Sports Medicine and Physical Fitness, 30(3), 268-275.

García-Pallarés, J., Sánchez-Medina, L., Carrasco, L., Díaz, A., $\&$ Izquierdo, M. (2009). Endurance and neuromuscular changes in worldclass level kayakers during a periodized training cycle. European Journal of Applied Physiology, 106, 629-638.

Gibala, M. J., Little, J. P., van Essen, M., Wilkin, G. P., Burgomaster, K. A., Safdar, A., Raha, S., \& Tarnopolsky, M. A. (2006). Short-term sprint interval versus traditional endurance training: similar initial adaptations in human skeletal muscle and exercise performance. Journal of Physiology, 15 (Pt 3), 901-911.

González-Badillo, J. J., Ribas-Serna, J. (2002). Bases de la programación del entrenamiento de la fuerza: Aplicación al alto rendimiento deportivo. Barcelona: INDE.

Gorostiaga, E. M., Granados, C., Ibáñez, J., \& Izquierdo, M. (2005). Differences in physical fitness and throwing velocity among elite and amateur male handball players. International Journal of Sports Medicine, 26(3), 225-232.

Helgerud, J., Høydal, K., Wang, E., Karlsen, T., Berg, P., Bjerkaas, M., Simonsen, T., Helgesen, C., Hjorth, N., Bach, R., \& Hoff, J. (2007). Aerobic high-intensity intervals improve VO2max more than moderate training. Medicine \& Science in Sports \& Exercise, 39(4), 665-671.

Impellizzeri, F. M., Rampinini, E., Maffiuletti, N., \& Marcora, S. M. (2007). A vertical jump force test for assessing bilateral strength asymmetry in athletes. Medicine \& Science in Sports \& Exercise, 39(11), 2044-2050.

Inbar, O., Bar-Or, O., \& Skinner, J. S. (1996). The Wingate
Anaerobic Test. Champaign, IL: Human Kinetics. Jeukendrup, A. E., Craig, N. P., \& Hawley, J. A. (2000). The bioenergetics of World Class Cycling. Journal of Science and Medicine in Sport, 3(4), 414-433.

Komi, P. V., \& Bosco, C. (1978). Utilization of stored elastic energy in leg extensor muscles by men and women. Medicine \& Science in Sports \& Exercise, 10(4), 261-265.

Laurent, C. M., Jr., Meyers, M.C., Robinson, C. A., \& Green, J. M. (2007). Cross-validation of the 20-versus 30-s Wingate anaerobic test. European Journal of Applied Physiology, 100(6), 645-651.

Lucia, A., Hoyos, J., Carvajal, A., \& Chicharro, J. L. (1999). Heart rate response to professional road cycling: the Tour de France. International Journal of Sports Medicine, 20(3), 167-172.

Lucia, A., Hoyos, J., \& Chicharro, J. L. (2001). Physiology of professional road cycling. Sports Medicine, 31(5), 325-337.

MacIntosh, B. R., Rishaug, P., \& Svedahl, K. (2003). Assessment of peak power and short-term work capacity. European Journal of Applied Physiology, 88(6), 572-529.

Marfell-Jones, M., Olds, T., Stewart, A. D., \& Carter, L. (2006). International Standards for Anthropometric Assessment. International Society for the Advancement of Kinanthropometry (ISAK): Potchefstroom, South Africa.

Martin, J. C., Wagner, B. M., \& Coyle, E. F. (1997). Inertialload method determines maximal cicling power in a single exercise bout. Medicine \& Science in Sports \& Exercise, 29, 1505-1512.

McIntyre, M. C., \& Hall M. (2005). Physiological profile in relation to playing position of elite college Gaelic footballers. British Journal of Sports Medicine, 39, 264-266.

Mujika, I., \& Padilla, S. (2001). Physiological and performance characteristics of male professional road cyclists. Sports Medicine, 31(7), 479-487.

Navarro, F., Verdugo, M. (2007). Módulo de Programación del entrenamiento de resistencia [Apuntes]. Madrid, España: Universidad Autónoma de Madrid: Máster de Alto Rendimiento del Comité Olímpico Español.

Neville, V., Pain, M. T., Kantor, J., \& Folland, J. P. (2010). Influence of crank length and crank-axle height on standing armcrank (grinding) power. Medicine \& Science in Sports \& Exercise, 42, 381-387.

Padilla, S., Mujika, I., Orbananos, J., Santisteban, J., Angulo, F., \& Jose Goiriena, J. (2001). Exercise intensity and load during massstart stage races in professional road cycling. Medicine \& Science in Sports \& Exercise, 33(5), 796-802.

Padilla, S., Mujika, I., Santisteban, J., Impellizzeri, F. M., \& Goiriena, J. J. (2008). Exercise intensity and load during uphill cycling in professional 3-week races. European Journal of Applied Physiology, 102(4), 431-438.

Pallarés, J. G., Morán-Navarro, R. (2012). Propuesta metodológica para el entrenamiento de la resistencia cardiorrespiratoria. Journal of Sport and Health Research. 4(2),119-136.

Riggs, M. P., \& Sheppard, J. M. (2009). The relative importance of strength and power qualities to vertical jump height of elite beach volleyball players during the counter-movement and squat jump. Journal of Human Sport and Exercise, 4(3), 221-236.

Rusko, H., Nummela, A., \& Mero, A. (1993). A new method for the evaluation of anaerobic running power in athletes. European Journal of Applied Physiology and Occupational Physiology, 66(2), 97-101.

Sands, W. A., McNeal, J. R., Ochi, M. T., Urbanek, T. L., Jemni, M., \& Stone, M. H. (2004). Comparison of the Wingate and Bosco anaerobic tests. Journal of Strength \& Conditioning Research, 18(4), 810-815.

Stacoff, A., Diezi, C., Luder, G., Stüsi, E., \& Krames-deQuervain, I.A. (2005). Ground reaction forces on stairs: effects of stair inclination and age. Gait \& Posture, 21, 24-38.

Szogy, A., \& Cherebetiu, G. (1974). A 1-min bicycle ergometer test for determination of anaerobic capacity. European Journal of Applied Physiology and Occupational Physiology, 33(2), 171-176.

Verchoschanskij, J. V. (1996). Topical problems of the modern theory and methodology of sports training. Coaching \& Sport 
Science Journal, 1(4), 2-10.

Vogt, S., Heinrich, L., Schumacher, Y. O., Blum, A., Roecker, K., Dickhuth, H. H., \& Schmid, A. (2006). Power output during stage racing in professional road cycling. Medicine \& Science in Sports \& Exercise, 38(1), 147-151.

Vogt, S., Schumacher, Y. O., Blum, A., Roecker, K., Dickhuth, H. H., Schmid, A \& Heinrich L. (2007). Cycling power output produced during flat and mountain stages in the Giro d'Italia: a case study. Journal of Sports Sciences, 25(12), 1299-1305.

Zabala, M., Peinado, A. B., Calderón, F. J., Sampedro, J., Castillo, M. J., \& Benito, P. J. (2011). Bicarbonate ingestion has no ergogenic effect on consecutive all out sprint tests in BMX elite cyclists. European Journal of Applied Physiology, 11(12), 3127-3134.

Zagatto, A. M., Papoti, M., \& Gobatto, C. A. (2008). Anaerobic capacity may not be determined by critical power model in elite table tennis players. Journal of Sports Science and Medicine, 7, 54-59. 